\title{
Access Point Implementation to Unifi Device with RADIUS and Captive Portal Authentication Method in PT XYZ
}

\author{
Halimatussa' diyah \\ Informatics and Computer Engineering \\ State Polytechnic of Jakarta \\ Depok, Indonesia \\ halimatussadiyah.tik15@pnj.ac.id
}

\begin{abstract}
The level of security at Local Area Network (LAN) that used today requires renewable technology. If the technology used is generally outdated and only uses a single password for protection, it is going to be easy to attacked by cyber criminals. Captive Portal is a static web page that force the user to login if want to use the network, it also needed for redirecting any user to specific partners website. RADIUS will take care for the validity from the user because RADIUS is an AAA Protocol (Authentication, Authorization, Accounting) for application like a network access or dynamic IP. RADIUS enables for server to filter anyone that want to enter the network. It will eliminates the vulnerability from the usage of network access and make the local area network more secure.
\end{abstract}

Keywords-security, local area network, password, radius, captive portal.

\section{INTRODUCTION}

Do you know that our society is still using static single password to connect to wifi? If it's, now is time to end that thing. One of any elements that critical for supporting business activities in provision increasing productivity is internet services. By utilizing the internet, the company will be facilitated in obtaining information effectively and efficiently. In the other hand the company can't be separated from the provision of internet networks for its employees, which of course is also a convenience and challenges that each company had. Local Area Network is the environment most used globally, but so many threat in the network at the same time. The threat must be handled and omitted before the user affected. Captive portal will redirecting any user want to use the LAN to login and RADIUS will check the validity of he given credentials that want to enter the network. If they don't have any credential or input the wrong credential they can't login to the network, the credential is not single password, it contains username or id card and password for sure. The Security of Local Area Network (LAN) has to be enhanced with RADIUS and Captive Portal because it can eliminates the vulnerability from the usage of network access and make local area network more secure.

\section{LITERATURE REVIEW}

Literature review needs to be done, because we need to review problems with the current situation in the community. Literature review can be done by looking at books, scientific journals from reliable publisher, and research results. The literature review discussed consists of the components below

\section{A. Unifi Controller Software}

Unifi Controller Software is a software from Unifi which is have to manage wireless networks, view network statistics with UniFi Controller Management Interface. Unifi Controller can also be used to manage wireless networks [7].

\section{B. PfSense}

PfSense software is free software, an open source distribution from the FreeBSD operating system that is specifically designed and to be used as a firewall and router that is fully managed through a web interface. PfSense in its use besides being a strong and flexible firewall and routing platform, it also includes a long list of related features and package systems that allow for further expansion without adding potential vulnerabilities security to the original distribution. The pfSense project was developed by Rubicon Communications [4].

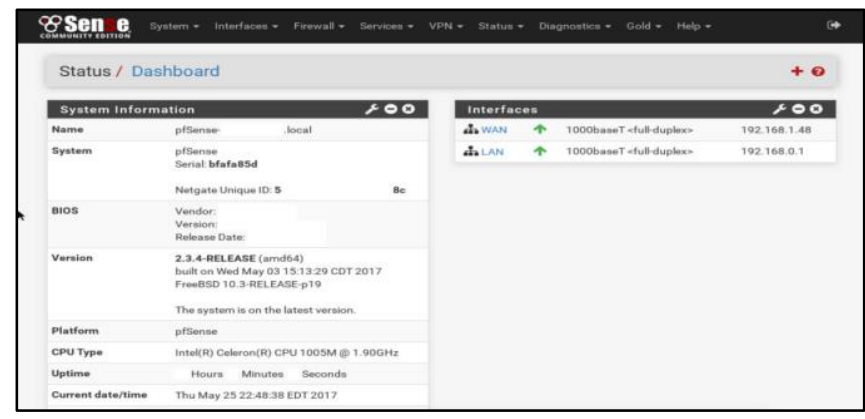

Fig. 1 PfSense panel website [5]

\section{RADIUS}

Remote Authentication Dial in User Service (RADIUS) is an AAA protocol (authentication, authorization, and accounting) for applications such as network access or IP mobility. RADIUS is usually used for network devices such as routers, modem servers, switches [9]. RADIUS is currently are standard for remote authentication and accounting. In RADIUS there are many vulnerability problems, these problems can be seen as security issues, transportation issues and implementation issues. RADIUS consistently provides several levels of protection against sniffing active attackers, but this indicates that the RADIUS protocol still has some leaks. This makes a lack of security for the RADIUS protocol [6].

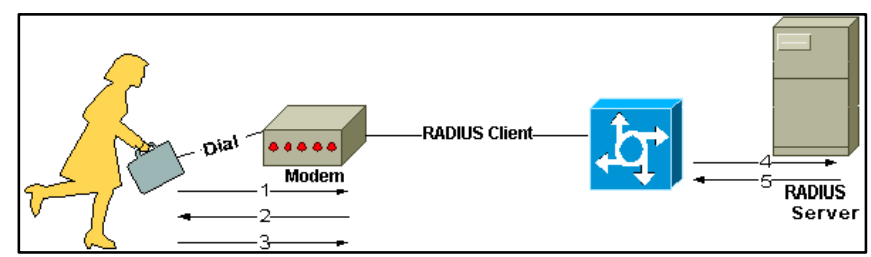

Fig. 2 How RADIUS works [2] 


\section{Captive Portal}

Captive Portal is a router or gateway device to protect any traffic, until the user registers to the system. Captive Portal is usually used in wireless infrastructure such as hotspots, but does not apply to wired networks. Captive portal works by diverting all requests for HTTP access from the user to a special page that is usually a user authentication page that contains questions such as a username and password or a page agreement between the user and provider. From a wireless network that functions to authenticate, before users access the internet. The http request switch is made by interrupting all packages and ignoring any addresses [8].

\section{E. Wide Area Network (WAN)}

Wide Area Network (WAN) is a network that has scope is satellite facilities, wireless or fiber optic cable because of its wider reach, even to other regions and countries[1].

\section{F. Local Area Network (LAN)}

Local Area Networks (LANs) are usually contained in one building, campus or geographical area, up to several kilometers. LANs are usually private and the main purpose is to connect computers and resources such as printers and data storage units belonging to a functional unit such as offices, factories, schools or universities. LANs are usually small, and LAN communication benefits from short delay times and reduced error rates. Data transmission rates range between 10 and $100 \mathrm{Mbps}$ with newer technologies reaching transmission speeds of up to $10 \mathrm{Gbps}$. The most popular technology for LAN is Ethernet, which is standard like IEEE 802.3[1].

\section{G. Authentication, Authorization, Accounting (AAA)}

Authentication is a process of verifying and validating the identity of a user before being allowed to access various resources such as devices, or services on a network. Authorization is the identification of definite variables followed by a certain level to allow rights or privileges to be identified for the person or object. Authorization is the process of granting permission to certain actions for certain entities that are in a global scenario specifically for users, devices, or applications. Whereas accounting is when a user has entered the network using various resources and services provided, the network administrator needs to make information tracks or commonly called logs. This log will enable network administrators to maintain, monitor user activities [9].

\section{H. Access Point}

Access Point receives data using an Ethernet cable, and converts it to $2.4 \mathrm{GHz}$ or $5 \mathrm{GHz}$ wireless signals. It will be useful to send and receive traffic from wireless networks to and from the client connected inside. Access points are different from wireless routers, access points do not have firewall functions, and access points will not protect the network from threats on the internet [3].

\section{METHOD}

The method used in this study is divided into several points, there are :

\section{A. Configure UNIFI Access Point}

In figure 3.1 site name is JTI The Plaza Office $16^{\text {th }}$. In configuration ssh authentication is enabled when admin want to remote access to the unifi controller.

\section{1) Country : For choosing country \\ 2) Timezone : For choosing timzeone from the country choosen \\ 3) LED Status : For turn on the led indicator in access point \\ 4) Uplink Connectivity : For select connectivity from the link}

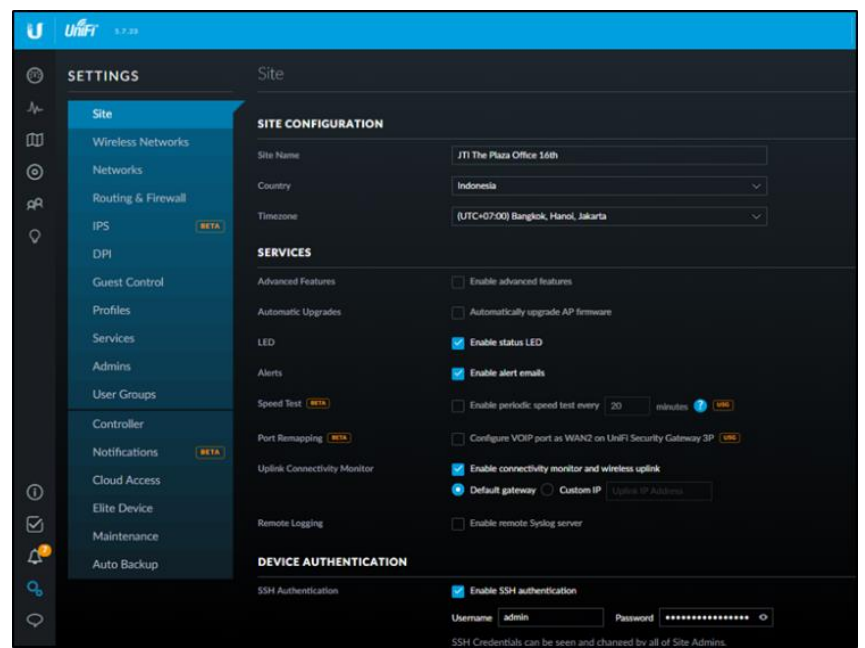

Fig. 3 Site Configuration

Figure 3.2 shows LAN configuration from Unifi. LAN has same gateway and subnet, it is 192.168.1.1/24. Figure 3.2 shows the specific configuration for local area network such as domain, gateway, purpose (description):
1) Purpose : For describing purpose of LAN
2) Domain :For choosing domain network
3) DHCP Mode : For choosing DHCP mode
4) DHCP NS : For give DHCP name server
5) DHCP Lease : For choosing lease time
6) $\mathrm{DHCP}$ gateway : For give gateway $\mathrm{DHCP}$

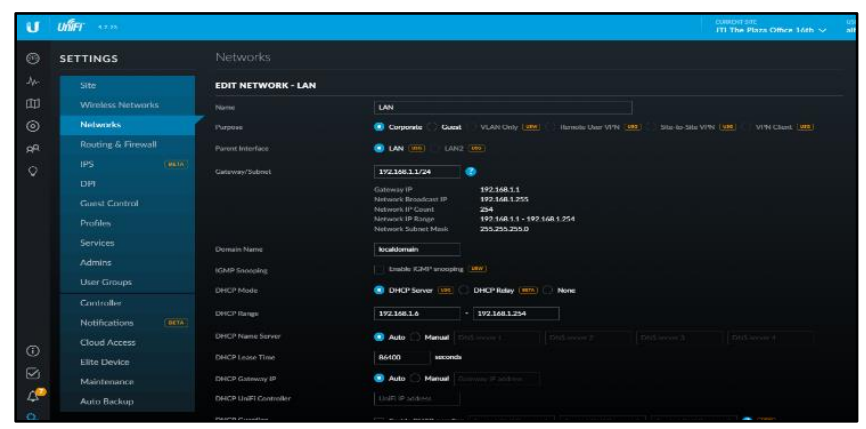

Fig. 4 LAN Configuration

Figure 3.3 shows the SSID that has been created, the name is WIFI_JTIGuest, it is open and the guest policies option is 
checked so that it can apply the captive portal later, along with an explanation :

\section{1) SSID Name : Name for SSID \\ 2) Enabled : Enabling SSID that want to create \\ 3) Security : : Authentication method \\ 4) Guest Policy : Activate captive portal}

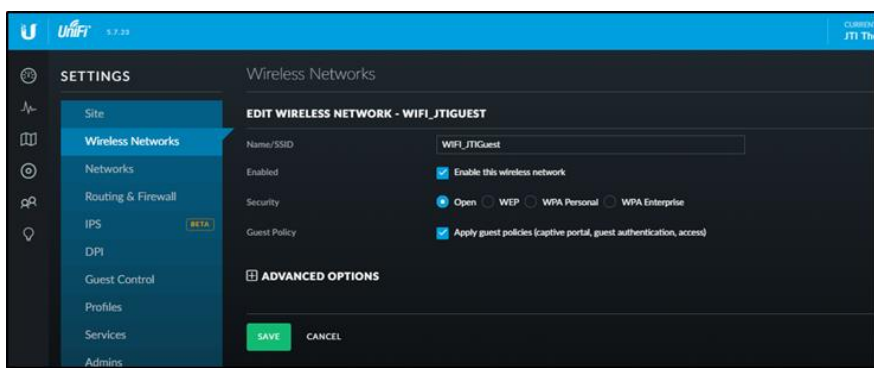

Fig. 5 SSID Configuration

\section{B. Configure RADIUS}

Figure 3.3 show RADIUS Profile that has been created with name UNIFI-RADIUS. RADIUS auth server is 192.168.0.24 with port is 1812 is as same as RADIUS accounting server (192.168.0.24) with different port 1813 :

1) Profile Name : For Specifies the name of the RADIUS profile that want to create

2) Radius Auth : Set the IP, Port, and Password needed by Unifi in order to recognize the RADIUS server in the user authentication stage

3) Radius Accounting : Set the IP, Port, and Password needed by Unifi in order to recognize the RADIUS server in the management stage of the user who has entered the network profile that want to create

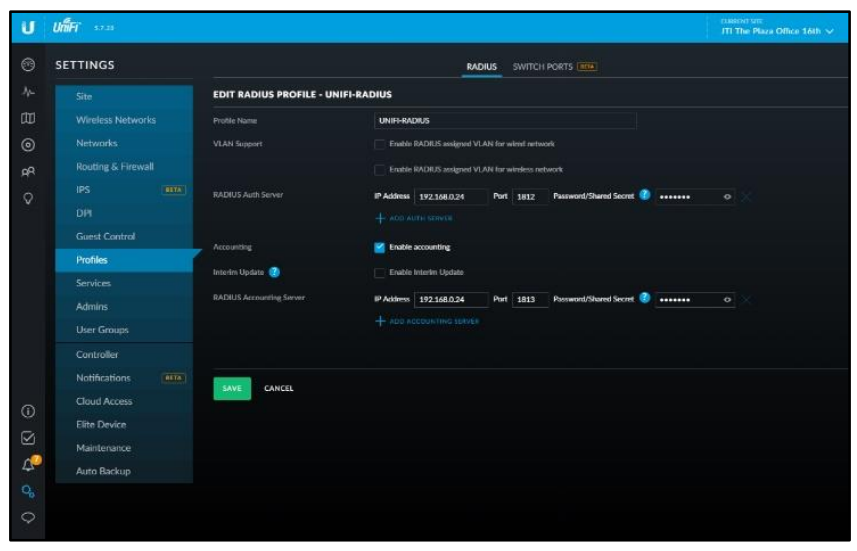

Fig. 6 RADIUS Configuration

\section{Configure Guest Access}

Figure 3.4 show the configuration for guest control, there are several parts, including :
1) Guest Portal
2) Authentication
3) Expiration
4) Landing Page

: For activate Captive Portal

: Auth method that want to use

: Default expiration date IP

: Landing page that want to use

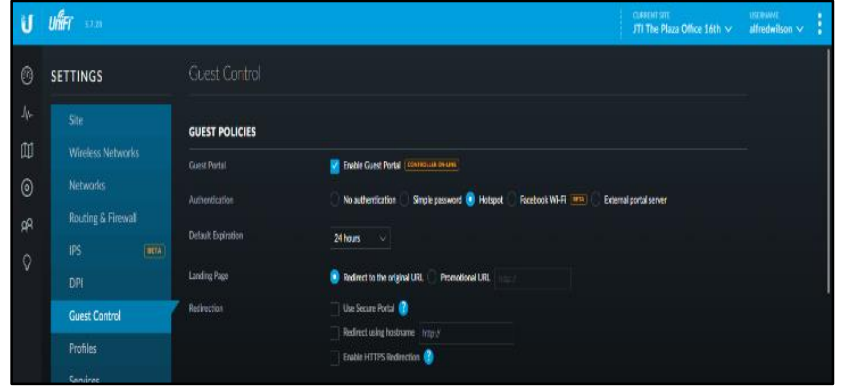

Fig. 7 Guest Configuration

\section{Generate Voucher User Guest Access}

Figure 3.5 there is a popup window to generate vouchers that will be used by the user using the guest access method. Vouchers made in the example use 10 vouchers, an expiration time of 24 hours and only applies to one user who can use it for 24 hours.

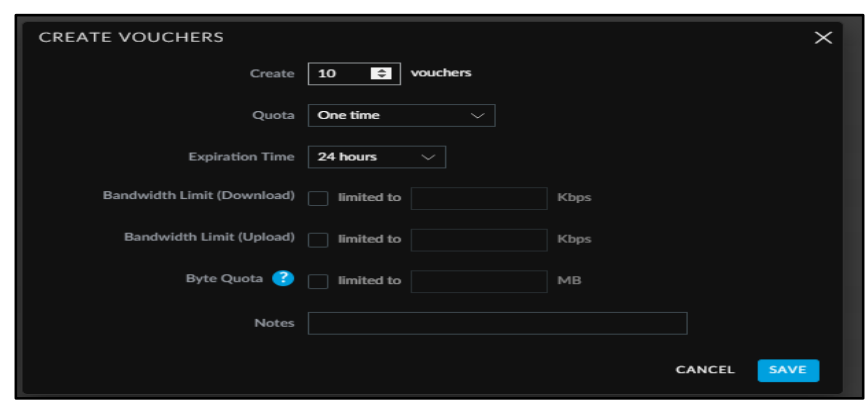

Fig. 8 Generate Vouchers

\section{E. Configure Bandwith Limit PfSense}

Figure 3.6 shows that the bandwidth coming from outside to pfsense will be set up to $2 \mathrm{Mbps}$ with an implementation schedule that is on the previously arranged OfficeHour schedule.

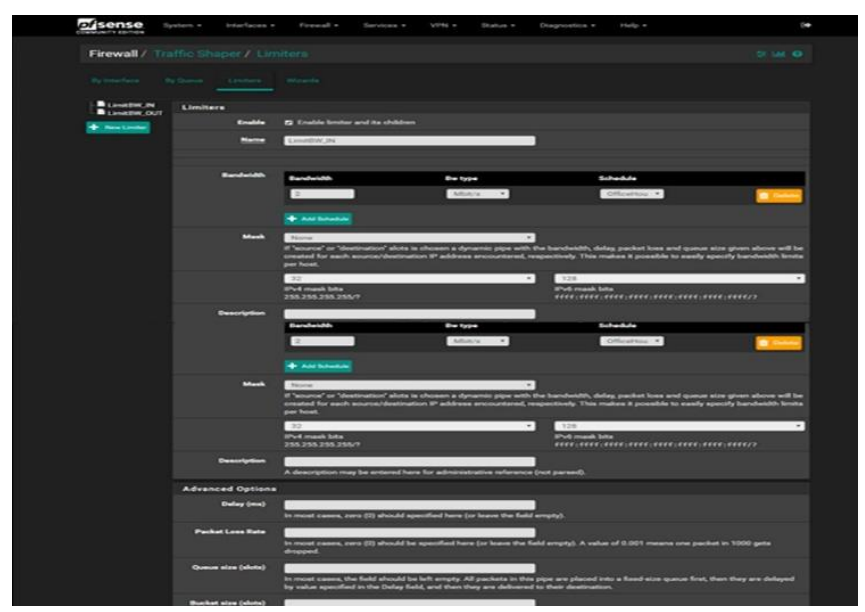

Fig. 9 Bandiwdth Limit

\section{F. Configure Firewall Schedule}

Figure 3.7 shows that the schedule for office hour is used for bandwidth limits. Schedule contains days, hours, schedule names and descriptions of schedules. 


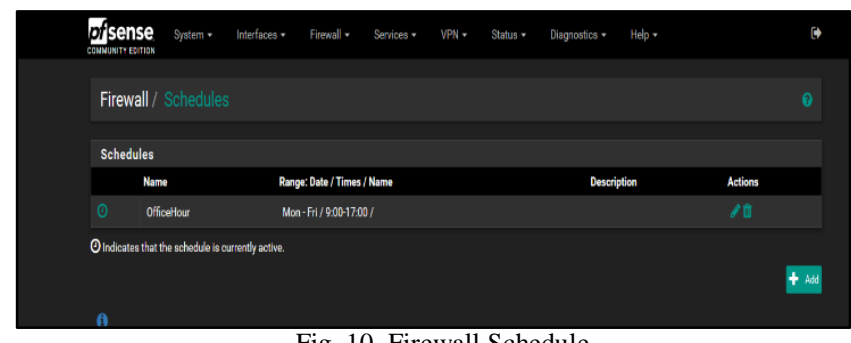

Fig. 10 Firewall Schedule

\section{RESULT}

Result from the system that has been created is when the system has passed the test and meet the initial requirements. Testing stage is by looking at the list of connected users, seeing the results of generating vouchers, testing vouchers to access the network, and testing using radius authentication for employees to enter the network

\section{A. Testing User List Connected}

To see users who are connected to access points with different methods.

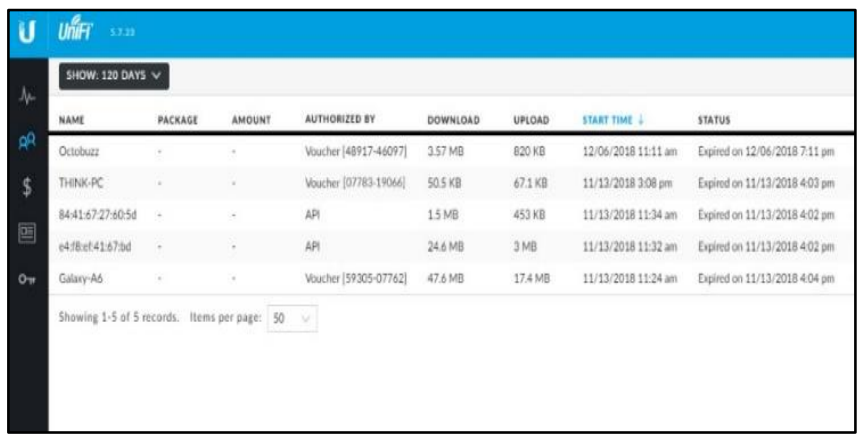

Fig. 11 User List Connected

The THINK-PC user is connected using a voucher code 07783-19066. In Figure 4.1 it can be concluded that the THINK-PC user has successfully connected to the access point that has been made because the voucher code used is appropriate, and the upload and download sessions has been started. Also user 84:41:67:60:5d connected using the RADIUS authentication method. In Figure 4.1 it can be concluded that user 84: 41: 67: 60: 5d has successfully connected to the access point that was created because it was successfully authorized, and the upload and download sessions have started.

\section{B. Testing Result from Generate Voucher}

The test is to see 10 voucher vouchers that have been made previously, in order to be used by users who do not have an ID and password as an employee. The voucher code that is created has a duration of 8 hours and can be used for one time use only.

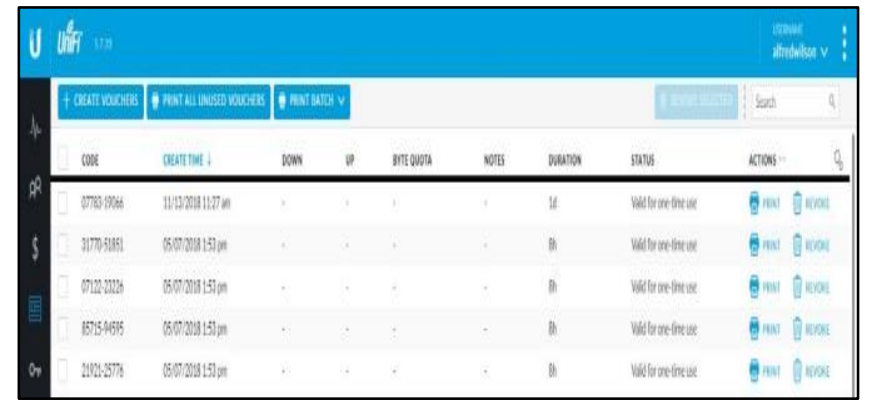

Fig. 12 List Generated Voucher

\section{Testing Voucher Access}

Making simulation method using a voucher (guest) with a voucher that was previously made and then tested for access to google.com. Testing of access vouchers is done using a voucher code 07783-19066.

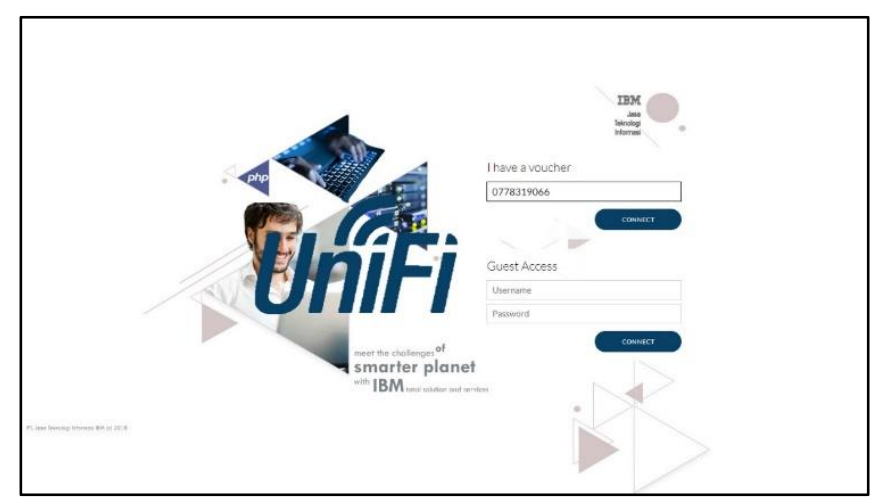

Fig. 13 Captive Portal Sign In

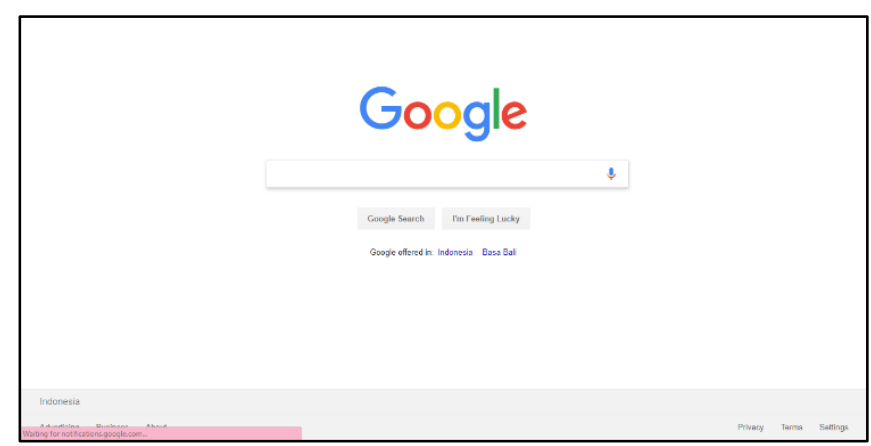

Fig. 14 Testing access google.com

\section{Testing RADIUS Access}

Making simulation method using the id and password (employee) that are in the active directory server and then access the google.com test, in figure 4.5 the username or id used is 902321 and r3ksoprodjo password. The RADIUS testing is different from voucher access because the RADIUS access there are only people who have registered on the radius server and have a username, password and authority to enter the network 


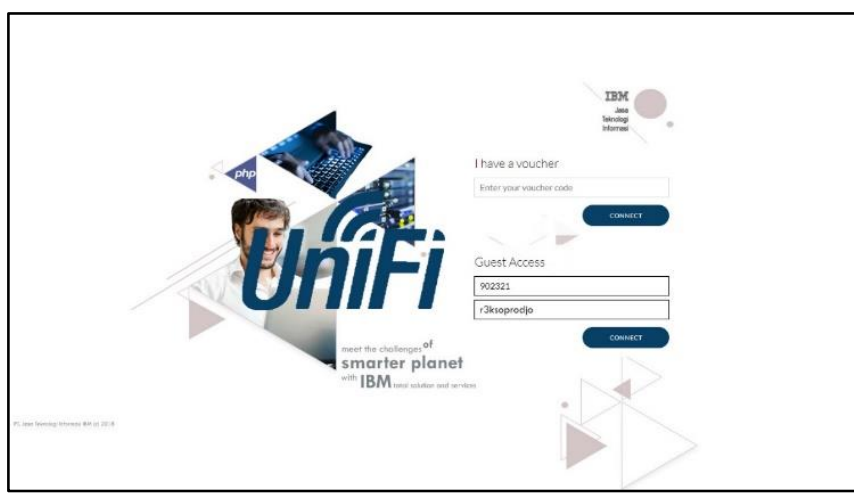

Fig. 15 Captive Portal Sign In

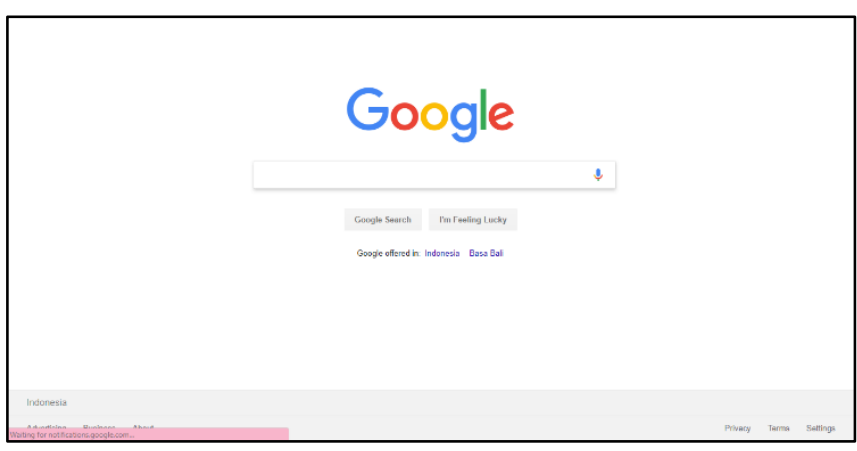

Fig. 16 Testing access google.com

\section{CONCLUSION}

The access point created is used when the user makes a request to enter the network and enter the captive portal, in which there are two access methods provided, using RADIUS which must use an employee credential that is integrated with the RADIUS server, and uses a voucher which is given. Apart from the authentication methods described, the network will not be accessible, so it is safe from unwanted attacks and network abuse. But it has limitation that sometimes unifi doesn't directly redirect user to the captive portal page and make the user can't login to the network.

\section{REFERENCES}

[1] B Ciubotaru, G.-M. M. (2014). Network Architectures. Advanced Network Programming - Principles and Techniques, Computer Communications and Networks, $3-28$.

[2] CISCO. (2018, 12 11). Remote Authentication Dial User Service (RADIUS). Retrieved from Cisco: https://www.cisco.com/c/en/us/support/docs/securityvpn/remote-authentication-dial-user-serviceradius/12433-32.html

[3] NETGEAR Support. (2018, Desember 11). What is a Wireless Access Point? Retrieved from NETGEAR: https://kb.netgear.com/235/What-is-a-wireless-accesspoint

[4] PFSense. (2018). About PFSense Features. Retrieved October 21, 2018, from https://www.pfsense.org/aboutpfsense/features.html

[5] PFSense. (2018, November 1). Learn About The PFSense. Retrieved from PFSense: https://www.pfsense.org/about-pfsense/
[6] Rehman, M. H., Govardhan, D. A., \& Narayana Rao, T. V. (2014). Design and Implementation of RADIUS An Network Security Protocol. Global Journal of Computer Science and Technology, 10(7), 48-54.

[7] Ubiquitti Networks. (2016). Unifi Controller User Guide. Ubiquitti Networks.

[8] Yutanto, H. (2018). Penerapan Model Promosi Berbasis Web Captive Portal Hotspot dengan Manajemen Terpusat. Journal Sistem Informasi Bisnis, 1(1), 49-56.

[9] Trnka, M., Cerny, T., \& Stickney, N. (2018). Survey of Authentication and Authorization for the Internet of Things. Hindawi Security and Communication Networks, 2018(2018), 1-18. 